\title{
Non-perturbative evaluation of $c_{\mathrm{SW}}$ for smeared link clover fermion and Iwasaki gauge action
}

\author{
Yusuke Taniguchi* \\ Graduate School of Pure and Applied Sciences, University of Tsukuba, \\ Tsukuba, Ibaraki 305-8571, Japan \\ E-mail: tanigchiehet.ph.tsukuba.ac.ip
}

\begin{abstract}
We performed a rough estimate of the non-perturbative value of the clover term coefficient $c_{\mathrm{SW}}$ for the APE stout link Wilson fermion. We varied the number of smearings from $N_{\text {smear }}=1$ to 6 and adopted $\beta$ values roughly corresponding to the lattice spacing of $0.1 \mathrm{fm}$. We used the Schrödinger functional technique for an evaluation of $c_{\mathrm{SW}}$ and found that $c_{\mathrm{SW}}$ decreases monotonically as we increase $N_{\text {smear }}$ but has a $10 \%$ order of deviation from the tree level value for $N_{\text {smear }}=6$.
\end{abstract}

The 30th International Symposium on Lattice Field Theory

June 24 - 29, 2012

Cairns, Australia

${ }^{*}$ Speaker. 


\section{Introduction}

The $O(a)$ improved Wilson fermion with the smeared link variable [ [ $\mathrm{d}, \mathrm{l}]$ ] is shown to have several virtues compared with the thin link fermion; a better scaling behavior, a fewer exceptional configuration [[]] and a better chiral behavior [四, 回].

The improvement factor $c_{\mathrm{SW}}$ for the clover term is expected to be smaller [ 6 ] than that for the unsmeared link action. The tree level tadpole improved value seems to be consistent with the

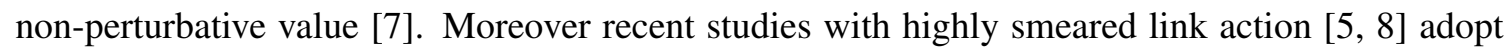
the tree level value $c_{\mathrm{SW}}=1$.

In this proceeding we would like to confirm if and how the non-perturbative value of $c_{\mathrm{SW}}$ is close to unity. We are also interested in the behavior of $c_{\mathrm{SW}}$ as we increase the number of smearings by fixing the lattice spacing. For this purpose we tried to set $\beta$ to correspond to the lattice spacing $a=0.1 \mathrm{fm}$.

\section{Schrödinger functional scheme}

For an evaluation of $c_{\mathrm{SW}}$ we make use of the well established Schrödinger functional technique [Q]. In this method we measure four kinds of two point functions between the axial current $A_{0}$ or the pseudo scalar density $P$ in the bulk and the "pseudo scalar density" $O(t=0)$ or $O^{\prime}(t=T)$ at the temporal boundary

$$
\begin{aligned}
& f_{A}\left(x_{0}\right)=-\frac{1}{N_{f}^{2}-1}\left\langle A_{0}^{a}\left(x_{0}\right) O^{a}\right\rangle, \quad f_{P}\left(x_{0}\right)=-\frac{1}{N_{f}^{2}-1}\left\langle P^{a}\left(x_{0}\right) O^{a}\right\rangle, \\
& f_{A}^{\prime}\left(x_{0}\right)=+\frac{1}{N_{f}^{2}-1}\left\langle A_{0}^{a}\left(T-x_{0}\right) O^{\prime a}\right\rangle, \quad f_{P}^{\prime}\left(x_{0}\right)=-\frac{1}{N_{f}^{2}-1}\left\langle P^{a}\left(T-x_{0}\right) O^{\prime a}\right\rangle .
\end{aligned}
$$

The PCAC quark masses are defined in terms of the improved current

$$
\begin{array}{ll}
m\left(x_{0}\right)=r\left(x_{0}\right)+c_{A} s\left(x_{0}\right), & m^{\prime}\left(x_{0}\right)=r^{\prime}\left(x_{0}\right)+c_{A} s^{\prime}\left(x_{0}\right), \\
r\left(x_{0}\right)=\frac{\left(\partial_{0}+\partial_{0}^{*}\right) f_{A}\left(x_{0}\right)}{4 f_{P}\left(x_{0}\right)}, & s\left(x_{0}\right)=\frac{a \partial_{0} \partial_{0}^{*} f_{P}\left(x_{0}\right)}{2 f_{P}\left(x_{0}\right)}, \\
r^{\prime}\left(x_{0}\right)=\frac{\left(\partial_{0}+\partial_{0}^{*}\right) f_{A}^{\prime}\left(x_{0}\right)}{4 f_{P}^{\prime}\left(x_{0}\right)}, & s^{\prime}\left(x_{0}\right)=\frac{a \partial_{0} \partial_{0}^{*} f_{P}^{\prime}\left(x_{0}\right)}{2 f_{P}^{\prime}\left(x_{0}\right)} .
\end{array}
$$

The improved factor $c_{A}$ for the axial current can be removed by adding an $O\left(a^{2}\right)$ term

$$
\begin{gathered}
M\left(x_{0}, y_{0}\right)=m\left(x_{0}\right)-\frac{m\left(y_{0}\right)-m^{\prime}\left(y_{0}\right)}{s\left(y_{0}\right)-s^{\prime}\left(y_{0}\right)} s\left(x_{0}\right)=r\left(x_{0}\right)-\frac{r\left(y_{0}\right)-r^{\prime}\left(y_{0}\right)}{s\left(y_{0}\right)-s^{\prime}\left(y_{0}\right)} s\left(x_{0}\right), \\
M^{\prime}\left(x_{0}, y_{0}\right)=m^{\prime}\left(x_{0}\right)-\frac{m^{\prime}\left(y_{0}\right)-m\left(y_{0}\right)}{s^{\prime}\left(y_{0}\right)-s\left(y_{0}\right)} s^{\prime}\left(x_{0}\right)=r^{\prime}\left(x_{0}\right)-\frac{r^{\prime}\left(y_{0}\right)-r\left(y_{0}\right)}{s^{\prime}\left(y_{0}\right)-s\left(y_{0}\right)} s^{\prime}\left(x_{0}\right) .
\end{gathered}
$$

The massless limit is given by tuning the hopping parameter so that

$$
M\left(\frac{T}{2}, \frac{T}{4}\right) \rightarrow 0
$$

and the non-perturbative $c_{\mathrm{SW}}$ is given by the improvement condition

$$
\Delta M=M\left(\frac{3 T}{4}, \frac{T}{4}\right)-M^{\prime}\left(\frac{3 T}{4}, \frac{T}{4}\right) \rightarrow 0
$$

with the hopping parameter set to its critical value $\kappa_{c}$. 


\section{Simulation setups}

We adopt the Iwasaki gauge action and the improved Wilson fermion action with the clover term. The number of flavors is set to $N_{f}=3$ with degenerate masses, which shall be tuned to zero. The APE stout smeared gauge link is used for those in the fermion action including the clover term. The smearing parameter is set to $\rho=0.1$ [四]. We vary the number of smearings from one to six. We adopt $8^{3} \times 16$ lattice with the Schrödinger functional boundary condition in the temporal direction [Q].

The gauge coupling $\beta$ is tuned so that the lattice spacing becomes around $0.1 \mathrm{fm}$. Since we would like to know a rough tendency of the improvement parameter $c_{\mathrm{SW}}$ we fix $\beta$ just by guess except for that at $N_{\text {smear }}=6$. The simulation parameter $\beta$ is given in table $\square$ for each $N_{\text {smear }}$ together with the inverse lattice spacing in the unit of $\mathrm{GeV}$. The inverse lattice spacing is measured on $24^{3} \times 48$ lattice by using the Sommer scale $r_{0}$ for $N_{\text {smear }}=1,2,3$ at unphysically heavy quark masses $m_{\pi}>500 \mathrm{MeV}$. Those at $N_{\text {smear }}=4,6$ are measured at the physical quark mass with the $\Omega$ baryon mass input.

The non-perturbative $c_{\mathrm{SW}}$ was not necessarily adopted for the measurement of $a^{-1}$. The $c_{\mathrm{SW}}$ used for the measurement is given in the fourth column of the table $\mathrm{W}$. As can be seen from the data at $N_{\text {smear }}=6$ the inverse lattice spacing $a^{-1}$ has a tendency to grow up when we increase $c_{\text {Sw }}$. The inverse lattice spacing given by $r_{0}$ also increase when we approach the physical quark mass point. $a^{-1}$ at $N_{\text {smear }}=1-4$ would appear to be larger than $2 \mathrm{GeV}$ with the non-perturbative $c_{\mathrm{SW}}$ at the physical quark mass.

Table 1: Number of smearings and $\beta$ for numerical simulation. The third column is a rough estimate of the lattice spacing measured with $c_{\mathrm{SW}}$ given in the fourth column. The data for $N_{\text {smear }}=0$ is taken from Ref. [四].

\begin{tabular}{|c|c|c|c|}
\hline$N_{\text {smear }}$ & $\beta$ & $a^{-1}(\mathrm{GeV})$ & $c_{\text {SW }}$ for $a^{-1}$ \\
\hline 0 & 1.90 & $2.194(10)$ & 1.715 \\
1 & 1.95 & 2.65 & 1.20 \\
2 & 1.93 & 2.35 & 1.00 \\
3 & 1.91 & 2.25 & 1.00 \\
4 & 1.89 & $2.119(88)$ & 1.00 \\
6 & 1.87 & $2.073(19)$ & 1.00 \\
6 & 1.87 & $2.340(26)$ & 1.10 \\
6 & 1.82 & $2.044(38)$ & 1.10 \\
6 & 1.82 & $2.192(09)$ & 1.13 \\
\hline
\end{tabular}

\section{Numerical results}

A typical behavior of the PCAC mass $M$ and the mass difference $\Delta M$ is plotted in figure $\square$ for $N_{\text {smear }}=6$ and $\beta=1.82$ at three values of $c_{\mathrm{SW}}$. The PCAC mass difference $\Delta M$ (up triangle) tends to decrease as we increase $c_{\mathrm{SW}}$ and crosses zero around $c_{\mathrm{SW}}=1.1$. 

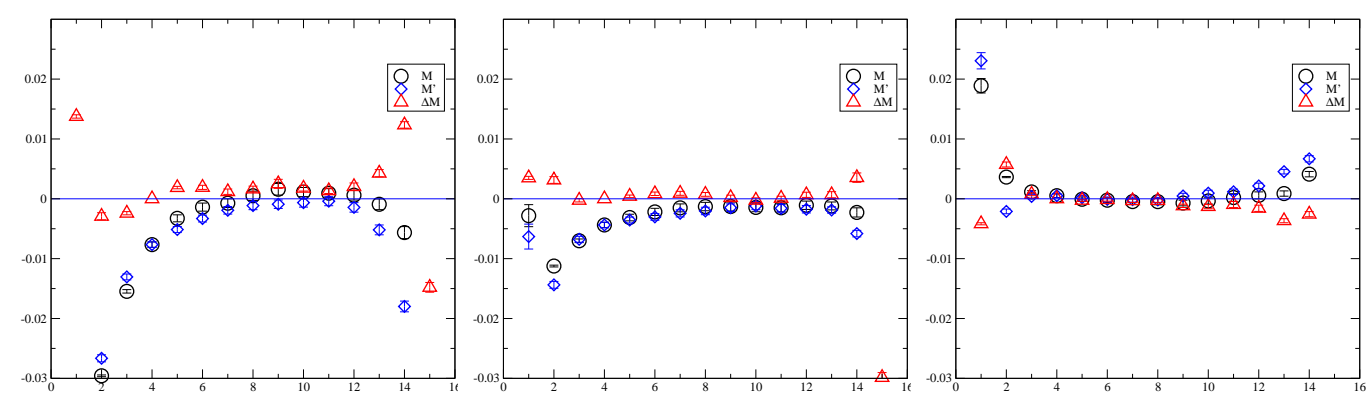

Figure 1: The PCAC mass $M\left(x_{0}, y_{0}\right), M^{\prime}\left(x_{0}, y_{0}\right)$ and the mass difference $\Delta M\left(x_{0}, y_{0}\right)$ as a function of $x_{0}$ for $N_{\text {smear }}=6, \beta=1.82 . y_{0}$ is set to $T / 4$. Three values of $c_{\mathrm{SW}}$ are adopted: 1.0 (left), 1.1 (middle) and 1.2 (right). The PCAC mass is tuned to be consistent with zero at $x_{0}=T / 2$.
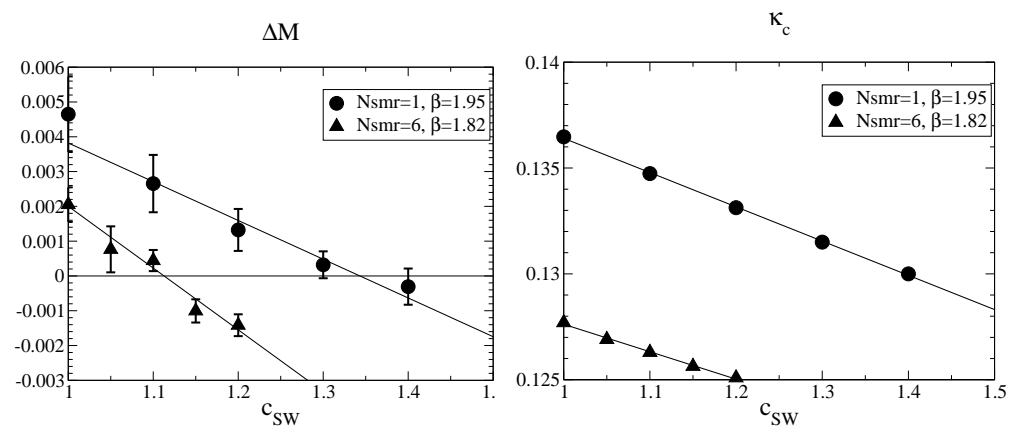

Figure 2: The PCAC mass difference $\Delta M(3 / 4 T, T / 4)$ (left) and the critical hopping parameter $\kappa_{c}$ (right) as a function of $c_{\mathrm{SW}}$. Two data are plotted with $N_{\text {smear }}=1$ (circle) and 6 (triangle).

We plot this behavior in the left panel of figure $\square$ for $N_{\text {smear }}=1$ and 6 . Both data can fitted by a linear function. The horizontal value where $\Delta M$ crosses zero is the non-perturbative $c_{\mathrm{SW}}$. The linear fit works well for other $N_{\text {smear }}$ and the result is listed in table $\square$.

Since the hopping parameter is tuned so that the PCAC mass is consistent with zero it represents the critical $\kappa_{c}$ at each $c_{\mathrm{SW}}$. $\kappa_{c}$ can also be fitted linearly as a function of $c_{\mathrm{SW}}$ as is shown in the right panel of figure $\square$. The value of $\kappa_{c}$ at the non-perturbative $c_{\mathrm{SW}}$ is listed in table $\square$ for each $N_{\text {smear }}$.

The non-perturbative $c_{\mathrm{SW}}$ is plotted as a function of number of smearings in the left panel of figure [3. $c_{\mathrm{SW}}$ decreases monotonically as a function of $N_{\text {smear }}$. We found roughly a $10 \%$ order of deviation from the tree level value even at $N_{\text {smear }}=6$. The critical hopping parameter $\kappa_{c}$ is also given in the right panel of figure [3. The decreasing behavior is almost the same as that of $c_{\mathrm{SW}}$ as a function of the number of smearings. $\kappa_{c}$ is very near to the tree level value $1 / 8$ at $N_{\text {smear }}=6$, which is one of the evidence of the good chiral behavior of the smeared link action.

From (12.6) and (2.7) a quantity

$$
c_{A}^{\prime}\left(x_{0}\right)=-\frac{r\left(x_{0}\right)-r^{\prime}\left(x_{0}\right)}{s\left(x_{0}\right)-s^{\prime}\left(x_{0}\right)}
$$

plays a role of the improvement coefficient of the axial vector current. As can be seen from figure 
Table 2: The result for the non-perturbative $c_{\mathrm{SW}}$ and the critical hopping parameter $\kappa_{c}$. The data for $N_{\text {smear }}=$ 0 is taken from Ref. [四].

\begin{tabular}{|c|c|c|c|}
\hline$N_{\text {smear }}$ & $\beta$ & $c_{\text {SW }}$ & $\kappa_{c}$ \\
\hline 0 & 1.90 & 1.715 & 0.13706 \\
1 & 1.95 & $1.342(21)$ & $0.13094(34)$ \\
2 & 1.93 & $1.187(18)$ & $0.12871(24)$ \\
3 & 1.91 & $1.155(43)$ & $0.12702(48)$ \\
4 & 1.89 & $1.137(19)$ & $0.12629(23)$ \\
6 & 1.87 & $1.057(20)$ & $0.12634(22)$ \\
6 & 1.82 & $1.1127(96)$ & $0.12612(16)$ \\
\hline
\end{tabular}
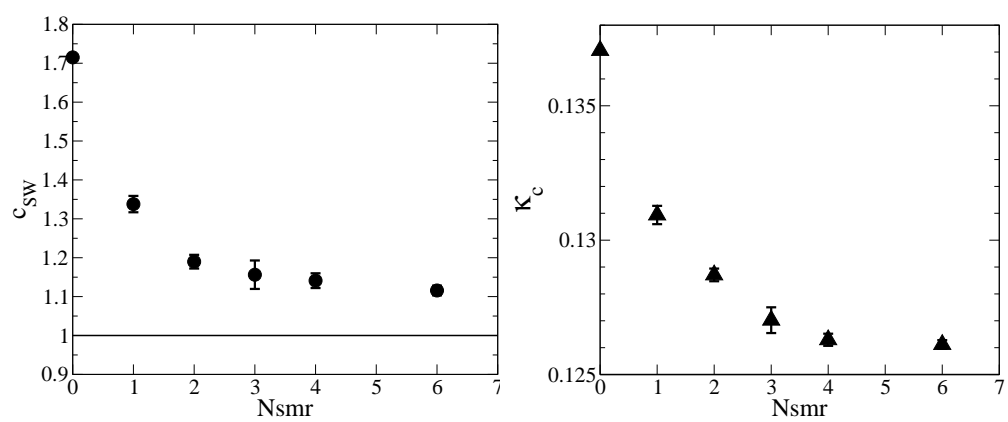

Figure 3: The non-perturbative $c_{\mathrm{SW}}$ (left panel) and the critical hopping parameter $\kappa_{c}$ (right panel) as a function of the number of smearings.

W $x_{0}$ dependence of $c_{A}^{\prime}$ is very flat near the non-perturbative $c_{\mathrm{SW}}$ and we are able to define the improvement factor of the axial current by

$$
c_{A}=c_{A}^{\prime}\left(\frac{T}{4}\right)
$$

This $c_{A}$ is fitted linearly as a function of $c_{\mathrm{SW}}$ as is shown in figure 5 . We evaluate $c_{A}$ at the nonperturbative $c_{\mathrm{SW}}$ and adopt it as its non-perturbative value. The results are plotted in figure 6 for each $N_{\text {smear }}$, which turned out to be very small and are consistent with zero within the statistical error.

\section{Conclusion}

We evaluate the non-perturbative value of the improvement coefficient $c_{\mathrm{SW}}$ of the clover term for the APE smeared link fermion action. We adopted $N_{\text {smear }}=1-6$ as the number of smearings. The bare coupling $\beta$ is tuned so that the lattice spacing is near to $a \sim 0.1 \mathrm{fm}$ as possible. The result is given in table $\square$ and figure [1]. $c_{\mathrm{SW}}$ decreases smoothly as we increase the number of smearings. However we found a $10 \%$ deviation from the tree level value even at $N_{\text {smear }}=6$.

As a byproduct we also evaluate the improvement factor $c_{A}$ of the axial current, which turned out to be consistent with zero within the statistical error. 

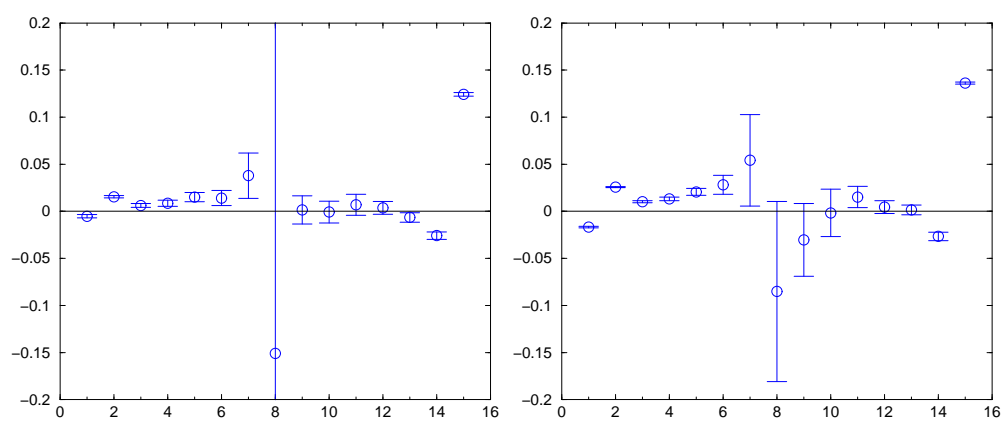

Figure 4: The axial current improvement factor $c_{A}^{\prime}$ as a function of $x_{0}$. The $c_{\mathrm{SW}}$ is set to be nearest to the non-perturbative value. The left panel is $N_{\mathrm{smear}}=1, c_{\mathrm{SW}}=1.3$. The right panel is $N_{\text {smear }}=6, c_{\mathrm{SW}}=1.1$. The hopping parameter is set to $\kappa_{c}$.

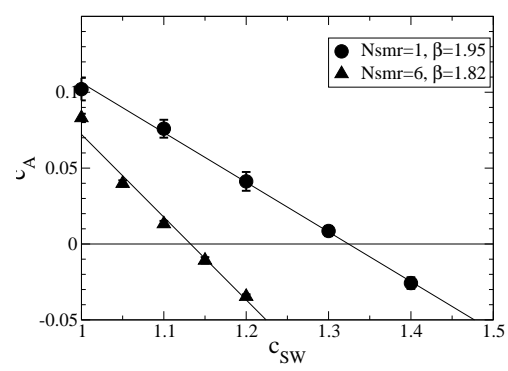

Figure 5: The improvement factor $c_{A}$ as a function of $c_{\mathrm{Sw}}$. Two data are plotted with $N_{\text {smear }}=1$ (circle) and 6 (triangle).

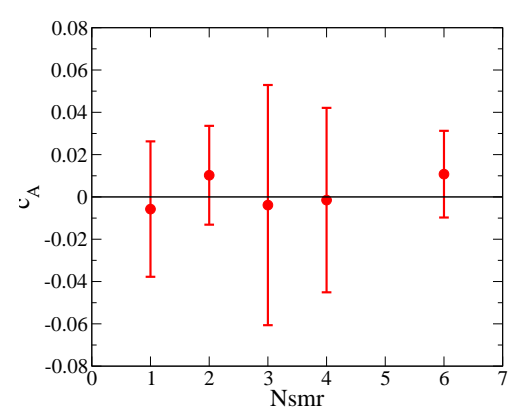

Figure 6: The non-perturbative improvement factor $c_{A}$ as a function of the number of smearings $N_{\text {smear }}$. All the data are consistent with zero within the statistical error.

\section{Acknowledgement}

This work is supported in part by Grants-in-Aid of the Ministry of Education (Nos. 22540265, 23105701).

\section{References}

[1] C. Morningstar and M. J. Peardon, Phys. Rev. D 69, 054501 (2004) [hep-lat/0311018]. 
[2] S. Capitani, S. Durr and C. Hoelbling, JHEP 0611, 028 (2006) [hep-lat/0607006].

[3] S. Durr, Z. Fodor, C. Hoelbling, R. Hoffmann, S. D. Katz, S. Krieg, T. Kurth and L. Lellouch et al., Phys. Rev. D 79, 014501 (2009) [arXiv:0802.2706 [hep-lat]].

[4] S. Capitani, S. Durr and C. Hoelbling, PoS LAT 2006, 157 (2006) [hep-lat/0609059].

[5] S. Durr, Z. Fodor, C. Hoelbling, S. D. Katz, S. Krieg, T. Kurth, L. Lellouch and T. Lippert et al., Phys. Lett. B 705, 477 (2011) [arXiv:1106.3230 [hep-lat]].

[6] R. Horsley, H. Perlt, A. Schiller, P. E. L. Rakow and G. Schierholz, PoS LAT 2007, 250 (2007) [arXiv:0710.0990 [hep-lat]].

[7] R. G. Edwards, B. Joo and H. -W. Lin, Phys. Rev. D 78, 054501 (2008) [arXiv:0803.3960 [hep-lat]].

[8] S. Durr, Z. Fodor, C. Hoelbling, S. D. Katz, S. Krieg, T. Kurth, L. Lellouch and T. Lippert et al., Phys. Lett. B 701, 265 (2011) [arXiv:1011.2403 [hep-lat]].

[9] M. Luscher, S. Sint, R. Sommer, P. Weisz and U. Wolff, Nucl. Phys. B 491 (1997) 323 [arXiv:hep-lat/9609035].

[10] S. Aoki et al. [PACS-CS Collaboration], Phys. Rev. D 81, 074503 (2010) [arXiv:0911.2561 [hep-lat]]. 